\title{
LA PLACE DES ÉCRIVAINES DANS LA LITTÉRATURE MONDIALE: CARTOGRAPHIE MÉTACRITIQUE DE LA MARGINALISATION DES FEMMES ${ }^{1}$
}

\begin{abstract}
Carolina Ferrer ${ }^{2}$
Résumé: Cette étude surgit de l'articulation du concept de champ (BOURDIEU, 1992) avec la scientométrie (PRICE, 1963). En nous basant sur l'approche de la criticométrie (FERRER, 2011), nous analysons empiriquement la réception critique des écrivaines et de leurs œuvres à partir de l'exploitation des références de la Modern Language Association International Bibliography. Ainsi, à travers l'analyse des métadonnées et l'élaboration de plusieurs cartes et indicateurs, couvrant la période 1844-2016, nous montrons que les écrivaines occupent une place minoritaire dans les publications. Elles sont nettement sous-représentées dans la majorité des littératures nationales, notamment en Europe et aux ÉtatsUnis. En particulier, l'analyse métacritique de la bibliographie sur 10 des écrivaines les plus étudiées dévoile une circulation plutôt restreinte de leurs œuvres. Ces constats nous permettent d'affirmer que les femmes occupent une place marginale dans les études littéraires.
\end{abstract}

Mots-clés: Place des écrivaines; littérature mondiale; criticométrie; marginalisation des femmes.

\section{EL LUGAR DE LAS ESCRITORAS EN LA LITERATURA MUNDIAL: CARTOGRAFÍA METACRÍTICA DE LA MARGINALIZACIÓN DE LAS MUJERES}

Resumen: Este estudio surge de la articulación del concepto de campo (BOURDIEU, 1992) con la cienciometría (PRICE, 1963). Basándonos en el enfoque de la criticometría (FERRER, 2011), analizamos empíricamente la recepción crítica de las escritoras y de sus obras gracias a la explotación de las referencias de la Modern Language Association International Bibliography. A través del análisis de los metadatos y de la elaboración de diversos mapas e indicadores, para el período 1844-2016, mostramos que las escritoras ocupan un lugar minoritario en las publicaciones y que están claramente sub-representadas en la mayoría de las literaturas nacionales, especialmente en Europa.

\footnotetext{
${ }^{1}$ Je tiens à remercier le Conseil de recherches en sciences humaines du Canada, qui finance notre projet intitulé « Les études littéraires et les nouveaux observables de l'ère numérique : le système de la littérature mondiale de l'après-guerre à nos jours », projet CRSH 435-2018-1115.

Professeure, Département d'études littéraires, Université du Québec à Montréal, ferrer.carolina@uqam.ca. Je tiens à remercier le Conseil de recherches en sciences humaines du Canada, qui finance le projet CRSH 435-2018-1115, intitulé « Les études littéraires et les nouveaux observables de l'ère numérique : le système de la littérature mondiale de l'après-guerre à nos jours », dans lequel s'inscrit cette étude. Endereço eletrônico: ferrer.carolina@uqam.ca
} 
Específicamente, el análisis metacrítico de la bibliografía sobre 10 de las escritoras más estudiadas revela una circulación relativamente restringida de sus obras. Estas constataciones nos permiten afirmar que las mujeres ocupan un lugar marginal en los estudios literarios.

Palabras clave: Lugar de las escritoras; literatura mundial; criticometría; marginalización de las mujeres.

Le film The Wife (2017) du réalisateur Björn Runge, adapté du roman de l'écrivaine étatsunienne Meg Wolitzer (2003), débute au moment où l'écrivain Joe Castleman reçoit un appel téléphonique de l'Académie suédoise lui annonçant qu'on vient de lui attribuer le prix Nobel de littérature. À travers de nombreux flashbacks, l'on découvre la complexe relation de Joe avec Joan, sa femme. En particulier, Joan se souvient du moment charnière où, alors qu'elle était jeune et passionnée par l'écriture, son futur mari lui présente l'écrivaine Elaine Mozell:

- Bonsoir, enchantée ! Votre prose est brillante. Elle est claire, elle est vivante et pleine d'audace - affirme la jeune femme.

- C'est très gentil. Malheureusement, le public déteste qu'une femme puisse publier une prose pleine d'audace... Il parait que vous avez du talent?

- Oh, merci, et oui, j'adore écrire, c'est vraiment toute ma vie.

- Ne faites pas ça !, lui lance Elaine.

- Qu'est-ce que vous dites?

- Vous voulez savoir où atterriront vos livres? Sur ces étagères, avec tous les bouquins des anciennes élèves. [Elaine prend un livre au hasard et le remet à Joan]. Ouvrez ce livre, par exemple. [le livre craque]. Vous entendez ça ? C'est le signe que jamais personne n'a emprunté ce bouquin. Vous devez comprendre que vous ne pouvez pas attirer leur attention.

- L'attention de qui ?, demande Joan.

- Des hommes. Ceux qui écrivent les critiques, les patrons des maisons d'édition, les rédacteurs en chef des magazines, tous ceux qui décident quel écrivain peut entrer dans la cour des grands, lequel mérite d'être mis sur un piédestal jusqu'à la fin de ses jours.

- Oui, mais un écrivain doit écrire ! insiste Joan.

- L'important, c'est qu'on vous lise ! Après cet avertissement, Elaine met fin à la conversation. 
Le long du film, nous découvrirons que Joan a bien compris le message d'Elaine, mais qu'elle a écrit quand même, en laissant son mari signer les livres. Ainsi, à Stockholm, au moment de la cérémonie de la remise du prix Nobel, elle est frappée par les paroles qui décrivent le style littéraire de Castleman: alors que son mari reçoit les honneurs, ce sont ses livres à elle qui sont décrits, c'est sa plume qui est mise en valeur. Évidemment, la cérémonie se traduit par une crise au sein du couple, mais la supercherie sera maintenue jusqu'après la mort de Joe. La question que nous voudrions retenir du film, c'est: dans quelle mesure le boys' club décrit par Elaine correspond-il à la réalité du champ littéraire?

Selon nos dernières recherches (FERRER, 2018), nous avons constaté un regain d'intérêt pour étudier la littérature à l'échelle planétaire, en intégrant le concept de mondialisation aux études littéraires (PRENDERGAST, 2004; SAUSSY, 2006; GUPTA, 2009; ZHANG, 2014). Cependant, cette ouverture d'esprit semble être accompagnée d'une tendance contraire par rapport aux écrivaines (PLANTÉ, 2003; UNDERWOOD and BAMMAN, 2016; GLORIEUX, 2017; LANGLAIS, 2017). En effet, des publications récentes soulignent un phénomène de "dé-féminisation» (LANGLAIS, 2017) de certaines activités, notamment la littérature. De plus, comme le signale Christine Planté (2003), il y aurait «un écart entre la présence de femmes écrivains dans la culture vécue et leur faible visibilité dans l'histoire littéraire» (p. 655). Ainsi, malgré les mouvements féministes survenus depuis l'après-guerre, les écrivaines continueraient de devoir surmonter de nombreux obstacles afin de faire rayonner leurs œuvres.

Afin de mieux comprendre la place de la littérature au féminin, dans cette étude, nous analysons la réception critique internationale des écrivaines.

\section{Le champ littéraire à la lumière de la criticométrie}

Selon Pierre Bourdieu, « le monde social moderne se décompose en une multitude de microcosmes, les champs, dont chacun possède des enjeux, des objets et des intérêts spécifiques » (BOURDIEU, 1997, p. 119). De plus, il met en lumière que des relations complexes se forment, non seulement entre les 
auteurs, mais aussi par rapport aux agents et aux institutions qui constituent le champ (BOURDIEU et WACQUANT, 1992, p. 72).

Par ailleurs, les interactions entre les différentes littératures nationales constituent aussi des phénomènes complexes, comme le signale la théorie des polysystémiques développée par Even-Zohar. Nous retenons que le terme "système » renvoie ici à des relations fonctionnelles ayant pour but l'élaboration d'hypothèses sur les phénomènes à l'étude. En fait, notre objectif principal est d'articuler des concepts théoriques; notamment celui de champ littéraire, avec la recherche empirique, afin de montrer la place qui revient aux écrivaines dans les études littéraires. Pour y parvenir, nous employons l'approche méthodologique de la criticométrie.

Initialement développée par Price (1963), l'objectif de la scientométrie est de mesurer et d'analyser l'activité dans les sciences et la technologie. Par analogie, nous avons appelé notre approche la criticométrie, puisqu'elle a pour objectif de mesurer et d'analyser l'activité critique en arts, et plus particulièrement en littérature. La méthode ici proposée correspond à l'adéquation des indicateurs scientométriques aux bases de données utilisées en sciences humaines et en arts.

Il existe deux catégories d'indicateurs scientométriques: les descriptifs et les relationnels. Les plus simples des indicateurs descriptifs sont le dénombrement des publications et le dénombrement des citations (LEYDESDORFF, 1998). Ce dernier correspond au nombre de fois qu'un texte est cité dans une autre publication ${ }^{3}$. Dans le cas de la criticométrie, nous retiendrons le point de vue de Kees van Rees, selon qui : «A reliable indicator of the quality attributed to a work of art is permanent and intensive attention -in the form of (spoken or written) discourses » (VAN REES, 1997, p. 93).

Par ailleurs, Henry Small (1973) a créé l'indicateur des cocitations. Il s'agit de comptabiliser le nombre de fois où deux références apparaissent simultanément dans les articles ${ }^{4}$. Nous constatons qu'en études littéraires il est essentiel de déterminer les types de relations que potentiellement

\footnotetext{
${ }^{3}$ En principe, cet indicateur signale la qualité d'une publication. Cependant, cet argument a été longuement débattu et il en résulte qu'il est préférable de le considérer comme un indicateur de visibilité (Cozzens 1985).

${ }^{4}$ Comme le souligne Loett Leydesdorff (1998), il est important de désagréger les différentes dimensions qui s'enchevêtrent dans les citations. Voir: Leydesdorff, «Theories of Citation?».
} 
entretiennent les différents éléments dans une citation. Ainsi, nous observons plusieurs couches de relations. Dans le cadre d'une publication critique, Tableau $n^{\circ} 1$, l'écrivain et l'œuvre littéraire analysés sont des objets d'étude pour l'auteur du document en question. En suivant van Rees, la référence constitue une reconnaissance de l'écrivain de la part du critique, alors que son texte établit une relation discursive avec l'œuvre analysée.

Par rapport aux écrivains qui sont cocités dans une publication critique, Tableau $\mathrm{n}^{\circ} 2$, ceux-ci peuvent entretenir une relation de prédécesseur/successeur ou être des auteurs contemporains. Dans tous les cas, ce sont des écrivains que le critique veut comparer en fonction d'une certaine thématique ou approche. Il en va de même pour les ouvrages cocités: ces textes sont l'objet d'une analyse comparée. De plus, les ouvrages littéraires cocités peuvent entretenir des relations que, en suivant Genette, nous appellerons transtextuelles ${ }^{5}$.

Avant d'analyser la place des écrivaines dans le champ littéraire planétaire, nous présentons les principaux résultats de nos recherches sur la littérature mondiale.

\begin{tabular}{|l|l|l|}
\hline \multicolumn{3}{|c|}{ Tableau $\mathbf{n}^{\circ} \mathbf{1}$} \\
\hline \multicolumn{3}{|c|}{ Relations entre la publication critique et l'œuvre littéraire } \\
\hline & Auteur citant & Publication critique \\
\hline Écrivain cité & Objet d'étude & Reconnaissance ou attention \\
\hline OEuvre citée & Objet d'étude & Relation discursive \\
\hline
\end{tabular}

\begin{tabular}{|l|l|l|}
\hline \multicolumn{3}{|c|}{${\text { Tableau } \mathbf{n}^{\circ} \mathbf{2}}^{\mid}$} \\
\hline \multicolumn{2}{|c|}{ Relations entre deux œuvres littéraires cocitées dans une publication critique } \\
\hline & Écrivain cité 2 & Euvre citée 2 \\
\hline Écrivain cité 1 & $\begin{array}{l}\text { Prédécesseur, contemporain, } \\
\text { successeur }\end{array}$ & $\begin{array}{l}\text { Source d'inspiration ou } \\
\text { d'influence }\end{array}$ \\
\hline Euvre citée 1 & $\begin{array}{l}\text { Source d'inspiration ou } \\
\text { d'influence }\end{array}$ & Relation inter/transtextuelle \\
\hline
\end{tabular}

\section{Cartographie de la littérature mondiale}

Afin d'obtenir les références par littérature nationale, nous avons interrogé la plus importante base littéraire, la Modern Language Association

\footnotetext{
${ }^{5}$ Selon Genette, «la transtextualité, ou transcendance textuelle du texte [...] [est] tout ce qui le met en
} relation, manifeste ou secrète, avec d'autres textes», Genette, Palimpsestes, p. 7. 
International Bibliography6, que désormais nous appellerons MLAIB. Cette base contient plus de 2,8 millions de références et couvre plus de 160 ans de publications effectuées par la communauté académique internationale. En utilisant les termes «littérature française», «littérature espagnole», et cæetera dans le champ «littérature nationale», nous avons obtenu les publications pour les 212 nations identifiées par les Nations Unies7. L'échantillon ainsi compilé s'élève à plus de 1,6 million de références et s'étend de 1844 à 20168.

La Carte $\mathrm{n}^{\circ} 1$ correspond au nombre de références par pays. Nous constatons qu'un très haut pourcentage des publications porte sur les littératures de certains pays européens et des États-Unis. En effet, si nous observons le Graphique $n^{\circ} 1$, nous constatons que les bibliographies critiques sur 14 littératures nationales cumulent plus de $82 \%$ des publications sur l'ensemble mondial. Le reste des littératures nationales se voient consacrer individuellement moins de $1 \%$ des publications.

Le Graphique $\mathrm{n}^{\circ} 2$ représente la distribution continentale des références cumulées. L'Europe concentre $69 \%$ des publications, alors que les Amériques détiennent $24 \%$ des références, l'Asie $4 \%$, l'Afrique $2 \%$ et l'Océanie $1 \%$

Le Tableau n ${ }^{\circ} 3$ correspond aux 20 écrivains les plus étudiés selon les références compilées9. Dans cette courte liste, il y a une seule femme, Virginia Woolf. Les 20 œuvres littéraires les plus analysées se trouvent sur le Tableau $n^{\circ} 4$. Il n'y a aucune femme parmi ces auteurs. Ces résultats indiquent très clairement que la plupart des publications critiques sont concentrées sur les littératures européennes et qu'elles sont biaisées vers des œuvres d'écrivains masculins anglophones.

\footnotetext{
${ }^{6}$ Modern Language Association International Bibliography. www.mla.org. Désormais, nous utiliserons I'acronyme MLAIB.

${ }^{7}$ Nous avons identifié plus de 200 nations pour l'année 2017, moment où nous avons interrogé MLAIB. Voir United Nations, http://data.un.org/Default.aspx.

${ }^{8}$ Dans cette étude, nous n'avons pas inclus les thèses doctorales, car elles proviennent exclusivement d'un répertoire étatsunien officiel, Dissertations Abstracts International, qui ne considère les thèses d'aucun autre pays. Voir http://proquest.com.

${ }^{9}$ Nous avons conservé l'orthographe des noms utilisée par MLAIB.
} 


\section{Carte $n^{\circ} 1$ : Bibliographie critique par littérature nationale}
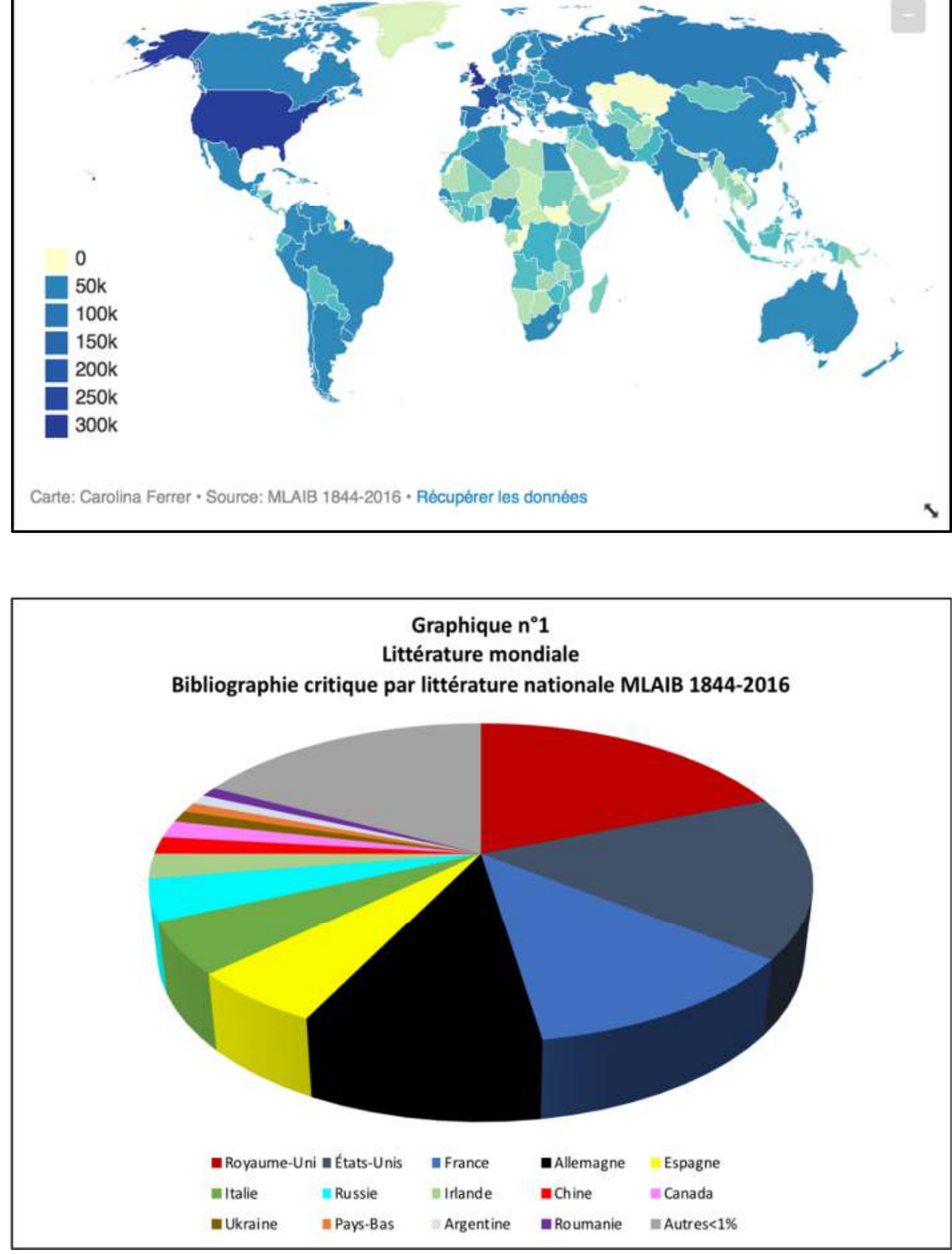


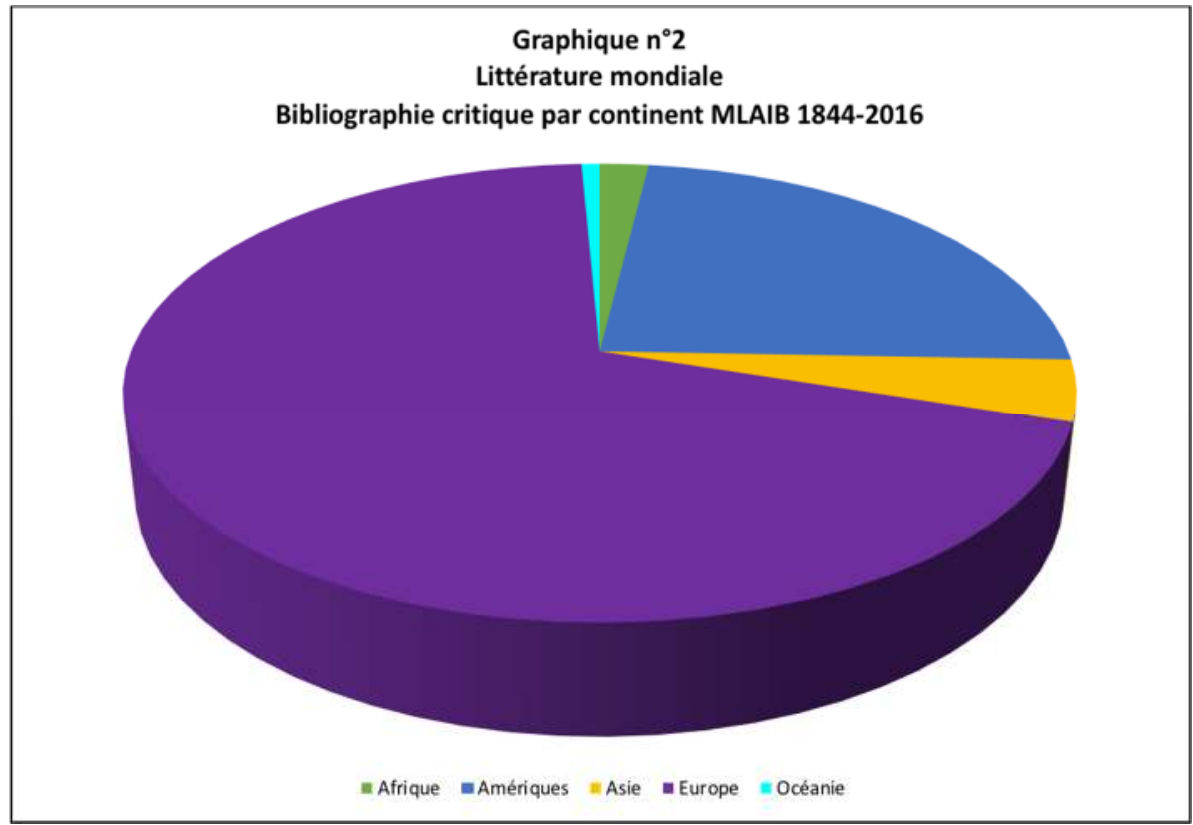

Tableau $\mathrm{n}^{\circ} 3$ : Les 20 écrivains les plus étudiés (MLAIB 1844-2016)

\begin{tabular}{|l|l|c|}
\hline Écrivain & Pays & $\%$ \\
\hline Shakespeare, William (1564-1616) & Royaume-Uni & $2,7 \%$ \\
\hline Dante (1265-1321) & Italie & $0,7 \%$ \\
\hline Joyce, James (1882-1941) & Irlande & $0,7 \%$ \\
\hline Goethe, Johann Wolfgang von (1749-1832) & Allemagne & $0,6 \%$ \\
\hline Chaucer, Geoffrey (1340/5-1400) & Royaume-Uni & $0,6 \%$ \\
\hline Milton, John (1608-1674) & Royaume-Uni & $0,5 \%$ \\
\hline Cervantes Saavedra, Miguel de (1547-1616) & Espagne & $0,4 \%$ \\
\hline Dickens, Charles (1812-1870) & Royaume-Uni & $0,4 \%$ \\
\hline Faulkner, William (1897-1962) & États-Unis & $0,4 \%$ \\
\hline James, Henry, Jr. (1843-1916) & États-Unis & $0,4 \%$ \\
\hline Beckett, Samuel (1906-1989) & Irlande & $0,4 \%$ \\
\hline Eliot, T. S. (1888-1965) & Royaume-Uni & $0,4 \%$ \\
\hline Melville, Herman (1819-1891) & États-Unis & $0,3 \%$ \\
\hline Woolf, Virginia (1882-1941) & Royaume-Uni & $\mathbf{0 , 3} \%$ \\
\hline Kafka, Franz (1883-1924) & République Tchèque & $0,3 \%$ \\
\hline Conrad, Joseph (1857-1924) & Royaume-Uni & $0,3 \%$ \\
\hline Wordsworth, William (1770-1850) & Royaume-Uni & $0,3 \%$ \\
\hline Nietzsche, Friedrich Wilhelm (1844-1900) & Allemagne & $0,3 \%$ \\
\hline Dostoevskiĭ, Fedor Mikhailovich (1821-1881) & Russie & $0,3 \%$ \\
\hline Hemingway, Ernest (1899-1961) & États-Unis & $0,3 \%$ \\
\hline & &
\end{tabular}


LA PLACE DES ÉCRIVAINES DANS LA LITTÉRATURE MONDIALE:

\begin{tabular}{|c|c|c|c|}
\hline \multicolumn{4}{|c|}{ Tableau n4 : Les 20 œuvres les plus étudiés (MLAIB 1844-2016) } \\
\hline OEuvre & Écrivain & Pays & $\%$ \\
\hline Hamlet $(1600-1601)$ & Shakespeare, William (1564-1616) & Royaume-Uni & $0,3 \%$ \\
\hline La Divina Commedia (ca. 1320) & Dante (1265-1321) & Italie & $0,2 \%$ \\
\hline Quijote $(1605,1615)$ & Cervantes Saavedra, Miguel de (1547-1616) & Espagne & $0,2 \%$ \\
\hline Ulysses (1922) & Joyce, James (1882-1941) & Irlande & $0,2 \%$ \\
\hline Paradise Lost (1667) & Milton, John (1608-1674) & Royaume-Uni & $0,2 \%$ \\
\hline Beowulf & n.n. & Royaume-Uni & $0,1 \%$ \\
\hline The Canterbury Tales & Chaucer, Geoffrey (1340/5-1400) & Royaume-Uni & $0,1 \%$ \\
\hline King Lear (1605-1606) & Shakespeare, William (1564-1616) & Royaume-Uni & $0,1 \%$ \\
\hline Othello (1604) & Shakespeare, William (1564-1616) & Royaume-Uni & $0,1 \%$ \\
\hline The Faerie Queene (1590-1596) & Spenser, Edmund (1552?-1599) & Royaume-Uni & $0,1 \%$ \\
\hline A la recherche du temps perdu (1913-1929) & Proust, Marcel (1871-1922) & France & $0,1 \%$ \\
\hline Essais (1580-1588) & Montaigne, Michel Eyquem de (1533-1592) & France & $0,1 \%$ \\
\hline Macbeth (1606) & Shakespeare, William (1564-1616) & Royaume-Uni & $0,1 \%$ \\
\hline The Tempest (1611) & Shakespeare, William (1564-1616) & Royaume-Uni & $0,1 \%$ \\
\hline Finnegans Wake (1939) & Joyce, James (1882-1941) & Irlande & $0,1 \%$ \\
\hline Faust $(1808,1832)$ & Goethe, Johann Wolfgang von (1749-1832) & Allemagne & $0,1 \%$ \\
\hline The Lord of the Rings trilogy & Tolkien, J. R. R. (1892-1973) & Royaume-Uni & $0,1 \%$ \\
\hline The Merchant of Venice (1596) & Shakespeare, William (1564-1616) & Royaume-Uni & $0,1 \%$ \\
\hline Piers Plowman & Langland, William & Royaume-Uni & $0,1 \%$ \\
\hline Moby-Dick (1851) & Melville, Herman (1819-1891) & États-Unis & $0,1 \%$ \\
\hline
\end{tabular}

\section{La littérature au féminin}

Afin de comparer le nombre d'hommes et de femmes qui sont l'objet d'étude des publications répertoriées dans MLAIB, nous avons créé un échantillon avec les écrivains dont la bibliographie critique est égale ou supérieure à $1 \%$ de la bibliographie sur la littérature nationale dans laquelle ils s'inscrivent. L'échantillon obtenu est de 1923 écrivains. Le Graphique $n^{\circ} 3$ correspond à leur répartition continentale. Au niveau mondial, les femmes représentent seulement $19 \%$ des écrivains. L'Europe présente le plus grand biais vers les auteurs masculins, avec $9 \%$ de femmes. Les Amériques détiennent le plus haut pourcentage de femmes: $27 \%$.

La Carte $\mathrm{n}^{\circ} 2$ correspond au pourcentage d'hommes dont la bibliographie critique concentre ou dépasse $1 \%$ des publications. Les pays en bleu foncé sont des littératures nationales où aucune femme n'a cumulé $1 \%$ de la bibliographie critique.

La Carte $\mathrm{n}^{\circ} 3$ représente le pourcentage de femmes dont la bibliographie critique est supérieure ou égale à $1 \%$ des publications sur la littérature nationale d'appartenance. À l'exception du Canada et de la Somalie, la pâleur de la carte reflète la basse présence des femmes parmi les écrivains étudiés. 


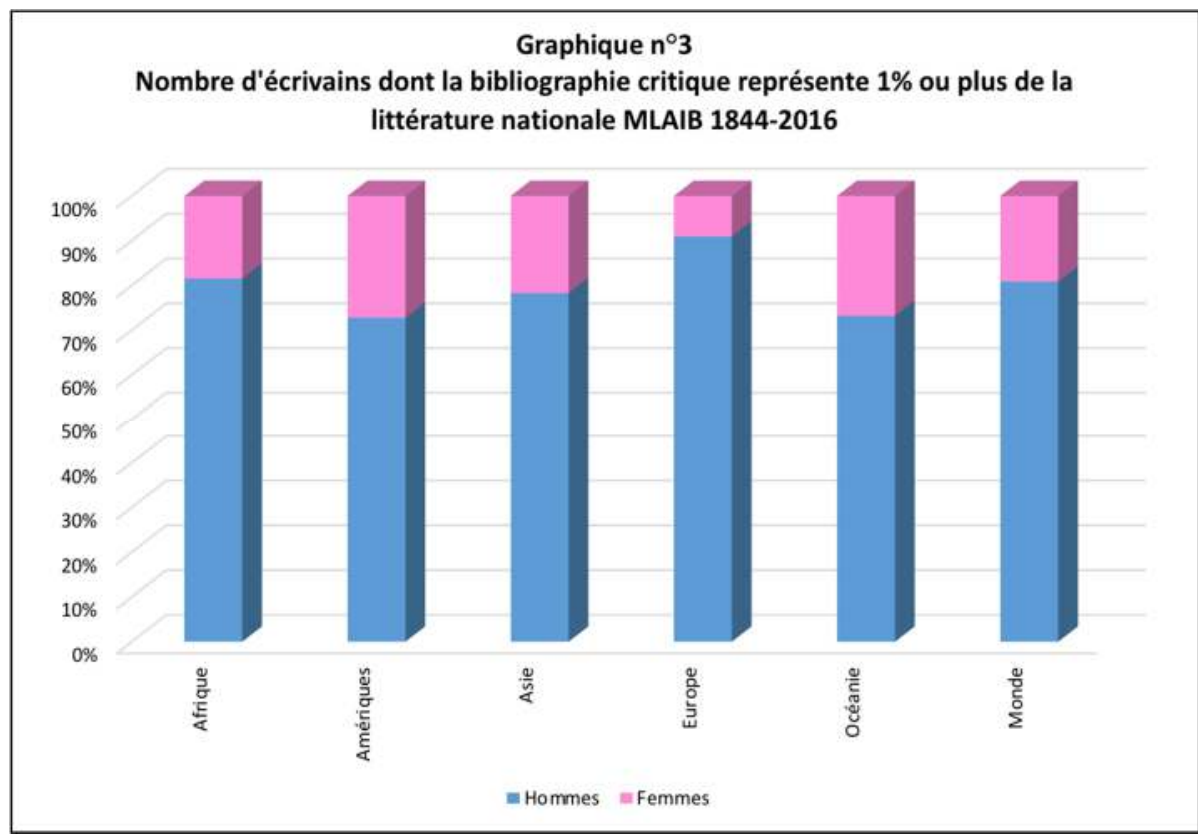

Carte $n^{\circ} 2$ : Hommes dont la bibliographie> $1 \%$ publications

$0 \%$

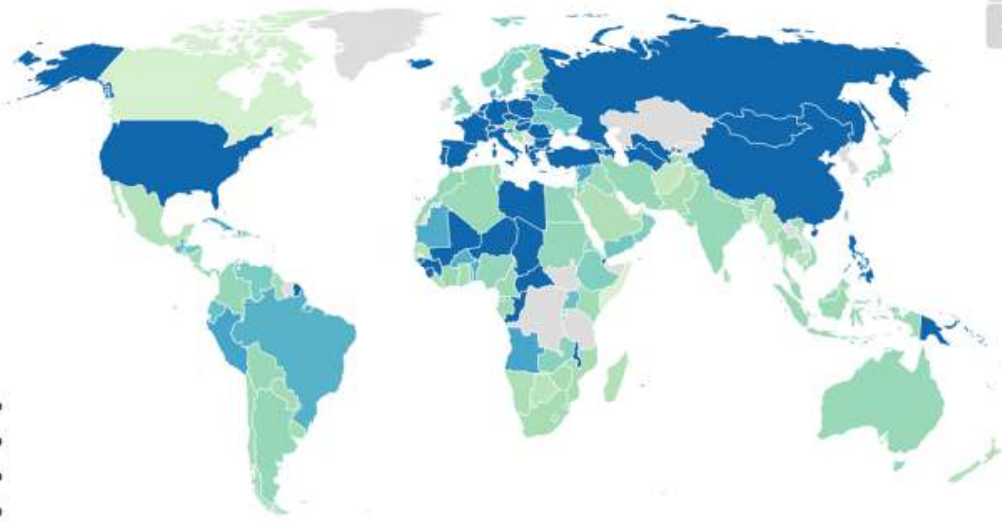

$80 \%$

$100 \%$ 


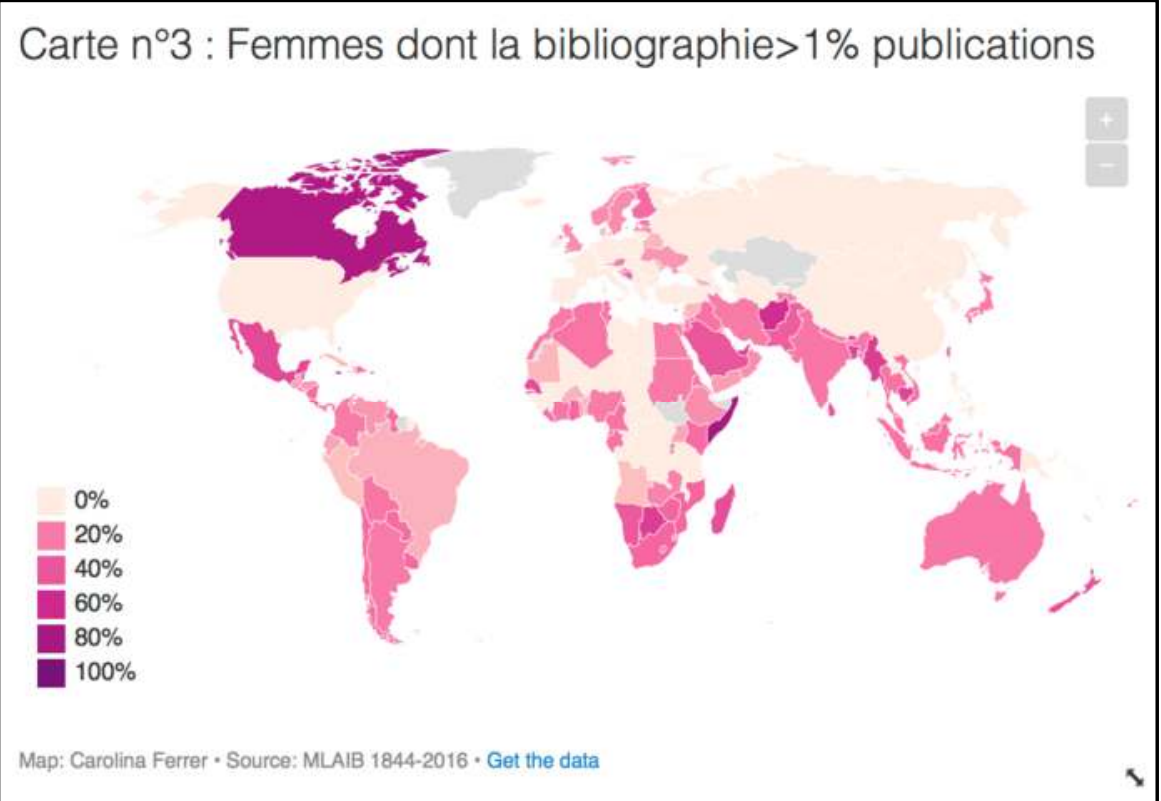

Le Tableau $n^{\circ} 5$ contient les 20 écrivaines qui, tout en représentant au moins $1 \%$ de la bibliographie sur leur littérature nationale, cumulent le plus grand nombre de références. Nous observons la présence de 4 Européennes, de 13 écrivaines des Amériques et de 3 Africaines. La plus ancienne de ces écrivaines est Juana Inés de la Cruz, qui vécut au 17e siècle en Nouvelle Espagne, région aujourd'hui connue comme le Mexique. La plus jeune est la Chilienne Isabel Allende.

Dans les tableaux suivants, nous présentons par continent les écrivaines qui, en plus de représenter au moins $1 \%$ de la bibliographie sur leur littérature nationale, cumulent 100 références critiques et plus. Avec 14 écrivaines, le Tableau $n^{\circ} 6$ correspond aux littératures africaines. Nous constatons la jeunesse de ces littératures: il y a une seule écrivaine du 19e siècle, alors que toutes les autres sont nées au 20e siècle. Nous observons aussi que 4 de ces auteures sont sud-africaines.

Le Tableau $n^{\circ} 7$ contient les 27 écrivaines des Amériques qui cumulent individuellement 100 références et plus. À l'exception de Juana Inés de la Cruz, déjà mentionnée, et de Mistral, Montgomery et Gómez de Avellaneda nées au 19e siècle, il s'agit d'écrivaines du 20e siècle. Tout comme l'Afrique, le champ littéraire des Amériques se démarque par sa jeunesse. 
Certaines littératures nationales montrent une présence plus importante, notamment le Canada avec 6 écrivaines, le Mexique avec 5, le Chili avec 4 et l'Argentine avec 3. Nous tenons à souligner qu'il n'y a aucune écrivaine étatsunienne sur cette liste, alors que les États-Unis constituent la deuxième littérature à niveau mondial selon les références répertoriées dans MLAIB.

Avec 2 écrivaines indiennes et 2 japonaises, le Tableau $n^{\circ} 8$ correspond au continent asiatique. Murasaki Shikibu a vécu au 10e siècle, mais les 3 autres sont nées au 20e siècle et sont encore en vie. Cette courte liste dévoile clairement la place exigüe réservée aux écrivaines dans le champ littéraire asiatique.

Le Tableau n ${ }^{\circ} 9$ correspond aux écrivaines du continent européen. La liste contient 8 noms, dont une écrivaine du 18e siècle, une du 20e siècle et 6 du 19e siècle. Alors que les littératures nationales européennes correspondent aux plus étudiées au monde, ce nombre extrêmement réduit d'écrivaines qui arrivent à attirer suffisamment l'attention de la critique est révélateur de l'étendue de la marginalisation des lettres au féminin qui a lieu en Europe.

Finalement, le Tableau $n^{\circ} 10$ contient les écrivaines de l'Océanie, dont une Néozélandaise et 2 Australiennes. Les trois sont du 20e siècle. Tout comme les cas de l'Afrique et des Amériques, le champ littéraire de l'Océanie se caractérise par sa jeunesse. Il faut souligner que, comparativement, la place des femmes y est beaucoup plus importante que celle qui leur est réservée dans les champs européen et asiatique, détenteurs d'une bien plus longue tradition.

À la lumière des données criticométriques, il est évident que, partout dans le monde, les écrivaines occupent une place marginale. Il nous semble assez surprenant que, dans les champs littéraires les plus développés, à savoir ceux de l'Europe et des États-Unis, les publications critiques qui portent sur les œuvres des femmes soient proportionnellement moins nombreuses que dans les champs littéraires en développement, c'est-à-dire ceux de l'Océanie, de l'Afrique et des Amériques à l'exception des États-Unis. 


\begin{tabular}{|l|l|l|r|}
\hline \multicolumn{5}{|c|}{ Tableau n`5 $\mathbf{~}^{\circ}$ Les 20 écrivaines les plus étudiées (MLAIB 1844-2016) } \\
\hline Continent & Pays & Écrivaine & $\%$ \\
\hline Europe & Royaume-Uni & Woolf, Virginia (1882-1941) & $1,7 \%$ \\
\hline Europe & Royaume-Uni & Austen, Jane (1775-1817) & $1,4 \%$ \\
\hline Amériques & Canada & Atwood, Margaret (1939-) & $4,4 \%$ \\
\hline Amériques & Mexique & Juana Inés de la Cruz (1648-1695) & $6,3 \%$ \\
\hline Amériques & Brésil & Lispector, Clarice (1924-1977) & $3,9 \%$ \\
\hline Europe & Ukraine & Ukraïnka, Lesia (1871-1913) & $2,7 \%$ \\
\hline Amériques & Canada & Munro, Alice (1931- $)$ & $1,7 \%$ \\
\hline Afrique & Afrique du Sud & Gordimer, Nadine (1923-2014) & $5,0 \%$ \\
\hline Afrique & Algérie & Djebar, Assia (1936-2015) & $25,6 \%$ \\
\hline Amériques & Chili & Mistral, Gabriela (1889-1957) & $6,3 \%$ \\
\hline Amériques & Chili & Allende, Isabel (1942- $)$ & $5,3 \%$ \\
\hline Amériques & Canada & Hébert, Anne (1916-2000) & $1,3 \%$ \\
\hline Amériques & Canada & Montgomery, L. M. (1874-1942) & $1,3 \%$ \\
\hline Amériques & Canada & Laurence, Margaret (1926-1987) & $1,2 \%$ \\
\hline Europe & Danemark & Blixen, Karen (1885-1962) & $4,6 \%$ \\
\hline Amériques & Canada & Roy, Gabrielle (1909-1983) & $1,2 \%$ \\
\hline Amériques & Mexique & Poniatowska, Elena (1933- ) & $2,1 \%$ \\
\hline Amériques & Mexique & Garro, Elena (1920-1998) & $1,9 \%$ \\
\hline Afrique & Afrique du Sud & Head, Bessie (1937-1986) & $3,0 \%$ \\
\hline Amériques & Mexique & Castellanos, Rosario (1925-1974) & $1,8 \%$ \\
\hline
\end{tabular}

\begin{tabular}{|l|l|r|}
\hline \multicolumn{2}{|c|}{ Tableau n'6 : Les écrivaines les plus étudiées de l'Afrique (MLAIB 1844-2016) } \\
\hline Pays & Écrivaine & $\%$ \\
\hline Afrique du Sud & Gordimer, Nadine (1923-2014) & $5,0 \%$ \\
\hline Algérie & Djebar, Assia (1936-2015) & $25,6 \%$ \\
\hline Afrique du Sud & Head, Bessie (1937-1986) & $3,0 \%$ \\
\hline Afrique du Sud & Schreiner, Olive Emilie Albertina (1855-1920) & $2,6 \%$ \\
\hline Nigeria & Emecheta, Buchi (1944-2017) & $3,7 \%$ \\
\hline Ghana & Aidoo, Ama Ata (1942- ) & $17,9 \%$ \\
\hline Sénégal & Bâ, Mariama (1929-1981) & $8,1 \%$ \\
\hline Nigeria & Adichie, Chimamanda Ngozi (1977-) & $2,2 \%$ \\
\hline Zimbabwe & Dangarembga, Tsitsi (1959- ) & $17,6 \%$ \\
\hline Algérie & Sebbar, Leilla (1941-) & $6,7 \%$ \\
\hline Nigeria & Nwapa, Flora (1931-1993) & $2,1 \%$ \\
\hline Zimbabwe & Vera, Yvonne (1964-2005) & $15,0 \%$ \\
\hline Cameroun & Beyala, Calixthe (1961- ) & $9,9 \%$ \\
\hline Afrique du Sud & Krog, Antjie (1952- ) & $1,1 \%$ \\
\hline
\end{tabular}




\begin{tabular}{|c|c|c|}
\hline Pays & Écrivaine & $\%$ \\
\hline Canada & Atwood, Margaret (1939-) & $4,4 \%$ \\
\hline Mexique & Juana Inés de la Cruz (1648-1695) & $6,3 \%$ \\
\hline Brésil & Lispector, Clarice (1924-1977) & $3,9 \%$ \\
\hline Canada & Munro, Alice (1931-) & $1,7 \%$ \\
\hline Chili & Mistral, Gabriela (1889-1957) & $6,3 \%$ \\
\hline Chili & Allende, Isabel (1942-) & $5,3 \%$ \\
\hline Canada & Hébert, Anne (1916-2000) & $1,3 \%$ \\
\hline Canada & Montgomery, L. M. (1874-1942) & $1,3 \%$ \\
\hline Canada & Laurence, Margaret (1926-1987) & $1,2 \%$ \\
\hline Canada & Roy, Gabrielle (1909-1983) & $1,2 \%$ \\
\hline Mexique & Poniatowska, Elena (1933-) & $2,1 \%$ \\
\hline Mexique & Garro, Elena (1920-1998) & $1,9 \%$ \\
\hline Mexique & Castellanos, Rosario (1925-1974) & $1,8 \%$ \\
\hline Cuba & Gómez de Avellaneda, Gertrudis (1814-1873) & $3,1 \%$ \\
\hline Argentine & Valenzuela, Luisa (1938-) & $1,5 \%$ \\
\hline Chili & Eltit, Diamela (1949- ) & $3,2 \%$ \\
\hline Uruguay & Peri Rossi, Cristina (1941-) & $7,3 \%$ \\
\hline Argentine & Pizarnik, Alejandra (1936-1972) & $1,1 \%$ \\
\hline Mexique & Boullosa, Carmen (1954- ) & $1,1 \%$ \\
\hline Argentine & Gambaro, Griselda (1928- ) & $1,0 \%$ \\
\hline Guatemala & Menchú, Rigoberta (1960?-) & $11,2 \%$ \\
\hline Porto Rico & Ferré, Rosario (1938-2016) & $6,1 \%$ \\
\hline Chili & Bombal, María Luisa (1910-1980) & $1,9 \%$ \\
\hline Trinité-et-Tobago & Brand, Dionne (1953-) & $8,6 \%$ \\
\hline Nicaragua & Belli, Gioconda (1948- ) & $6,0 \%$ \\
\hline Costa Rica & Vallbona, Rima de (1931-) & $12,5 \%$ \\
\hline Jamaïque & Cliff, Michelle (1946-) & $13,5 \%$ \\
\hline
\end{tabular}

\begin{tabular}{|l|l|r|}
\hline \multicolumn{2}{|c|}{ Tableau n ${ }^{\circ} 8:$ Les écrivaines les plus étudiées de l'Asie (MLAIB 1844-2016) } \\
\hline Pays & Écrivaine & $\%$ \\
\hline Japon & Murasaki Shikibu (fl. ca. 978-1020) & $2,4 \%$ \\
\hline Inde & Desai, Anita (1937-) & $1,6 \%$ \\
\hline Inde & Roy, Arundhati (1961- ) & $1,3 \%$ \\
\hline Japon & Tawada Yoko (1960- ) & $1,3 \%$ \\
\hline
\end{tabular}

\begin{tabular}{|l|l|r|}
\hline \multicolumn{2}{|c|}{ Tableau n'9 : Les écrivaines les plus étudiées de l'Europe (MLAIB 1844-2016) } \\
\hline Pays & Écrivaine & $\%$ \\
\hline Royaume-Uni & Woolf, Virginia (1882-1941) & $1,7 \%$ \\
\hline Royaume-Uni & Austen, Jane (1775-1817) & $1,4 \%$ \\
\hline Ukraine & Ukraïnka, Lesia (1871-1913) & $2,7 \%$ \\
\hline Danemark & Blixen, Karen (1885-1962) & $4,6 \%$ \\
\hline Suède & Lagerlöf, Selma (1858-1940) & $2,1 \%$ \\
\hline Lettonie & Pliekšāne, Elza (1865-1943) & $2,2 \%$ \\
\hline Finlande & Södergran, Edith Irene (1892-1923) & $4,4 \%$ \\
\hline Suède & Lindgren, Astrid Anna Emilia (1907-2002) & $1,3 \%$ \\
\hline
\end{tabular}


LA PLACE DES ÉCRIVAINES DANS LA LITTÉRATURE MONDIALE:

Tableau $\mathrm{n}^{\circ} 10$ : Les écrivaines les plus étudiées de l'Océanie (MLAIB 1844-2016)

\begin{tabular}{|l|l|r|}
\hline Pays & Écrivaine & \multicolumn{1}{c|}{$\%$} \\
\hline Nouvelle Zélande & Frame, Janet (1924-2004) & $11,3 \%$ \\
\hline Australie & Stead, Christina (1902-1983) & $1,8 \%$ \\
\hline Australie & Wright, Judith (1915-2000) & $1,1 \%$ \\
\hline
\end{tabular}

\section{Analyse métacritique des écrivaines les plus citées}

Afin de mieux comprendre la réception critique des femmes, nous avons sélectionné 10 écrivaines et avons élaboré une série d'indicateurs à partir des métadonnées des publications qui portent sur elles. L'échantillon est constitué par au moins une écrivaine par continent, tout en essayant d'y inclure une diversité de langues d'écriture. Ainsi, nous avons interrogé la base MLAIB à nouveau, en utilisant le nom de chaque écrivaine dans le champ "Author as subject». Dans le Tableau $n^{\circ} 11$, nous avons répertorié des informations de base à leur sujet. L'année de la première publication inscrite dans MLAIB nous permet de constater la jeunesse du corpus critique. L'écrivaine la plus ancienne de l'échantillon est Murasaki Shikibu du Japon, qui vécut vers la fin du premier millénaire. Cependant, il faut attendre jusqu'en 1940 pour voir paraître dans MLAIB des références sur son œuvre. Vient ensuite Sor Juana Inés de la Cruz, figure littéraire emblématique de la Nouvelle Espagne. Dans la base MLAIB, les publications sur son œuvre débutent en 1926. Pratiquement méconnue à l'extérieur de l'Ukraine, l'œuvre de Lesia Ukraïnka a certainement attiré l'attention de la critique nationale. Cependant, selon MLAIB, les références sur son œuvre commencent presque 70 ans après son décès. Concernant les publications critiques sur les 7 autres écrivaines sélectionnées, celles-ci commencent à paraître dans MLAIB de leur vivant.

\begin{tabular}{|c|c|c|c|c|c|}
\hline \multicolumn{6}{|c|}{ Tableau n 11 : Indicateurs métacritiques des écrivaines sélectionnées } \\
\hline Écrivaine & Pays & Période de vie & $\begin{array}{l}\text { Langue } \\
\text { d'écriture }\end{array}$ & Références & $1^{\mathrm{e}}$ réf. MLAIB \\
\hline Virginia Woolf & Royaume-Uni & $1882-1941$ & Anglais & 5159 & 1931 \\
\hline Juana Inés de la Cruz & Mexique (N. Espagne) & $1648-1695$ & Espagnol & 987 & 1926 \\
\hline Clarice Lispector & Brésil (Ukraine) & 1924-1977 & Portugais & 559 & 1967 \\
\hline Lesia Ukraïnka & Ukraine & $1871-1913$ & Ukrainien & 546 & 1981 \\
\hline Nadine Gordimer & Afrique du Sud & 1923-2014 & Anglais & 479 & 1981 \\
\hline Assia Djebar & Algérie & $1936-2015$ & Français & 476 & 1983 \\
\hline Karen Blixen & Danemark & $1885-1962$ & Anglais et danois & 369 & 1956 \\
\hline Murasaki Shikibu & Japon & $978 ?-1020 ?$ & Japonais & 281 & 1940 \\
\hline Anita Desai & Inde & $1937-$ & Anglais & 241 & 1969 \\
\hline Janet Frame & Nouvelle-Zélande & 1924-2004 & Anglais & 219 & 1965 \\
\hline
\end{tabular}


Nous avons représenté graphiquement les derniers indicateurs sur les écrivaines sélectionnées. Le Graphique $n^{\circ} 4$ correspond à la distribution linguistique de la bibliographie critique sur chaque écrivaine. L'œuvre de Woolf, Gordimer, Blixen, Shikibu, Desai et Frame ont été l'objet de publications essentiellement en anglais. Parmi celles-ci, la bibliographie la plus multilingue est celle qui porte sur Karen Blixen, ce qui reflète la réception de son œuvre non seulement au Danemark, mais dans toute la Scandinavie. Dans le cas de Shikibu, il y a aussi une importante réception locale, avec $21 \%$ des publications en japonais. Une proportion significative de la bibliographie sur Djebar est en français. Les bibliographies sur De la Cruz et sur Ukraïnka correspondent majoritairement à des textes publiés dans la langue d'écriture des auteures. Ces résultats sont des indicateurs assez évidents de la circulation plutôt restreinte de l'œuvre des écrivaines en question. La bibliographie sur Lispector est la plus diversifiée de l'échantillon.

Concernant les cocitations, le Graphique $n^{\circ} 5$ indique la littérature nationale d'appartenance des auteurs cocités qui représentent au moins $1 \%$ des références critiques de chaque auteur. Immédiatement, nous constatons que presque dans tous les cas, les écrivaines sont comparées à des compatriotes. Ainsi, pour ce sous-ensemble, nous pouvons affirmer que la majorité des études comparées sont de nature nationale. Les seules exceptions sont, d'une part, Djebar, dont les cocitations sont équitablement réparties entre Algériens et Français, cependant, il ne faut pas perdre de vue que le principal auteur français cocité est Albert Camus, lui aussi né en Algérie. D’autre part, les cocitations de l'œuvre de Juana Inés de la Cruz sont principalement divisées entre les littératures mexicaine et espagnole, situation qui s'explique facilement, car elle est très souvent mise en relation avec des écrivains du Siècle d'or espagnol.

Lorsque nous représentons les cocitations au niveau continental, Graphique $n^{\circ} 6$, nous observons à quel point les cocitations renvoient au continent d'appartenance de l'écrivain. En même temps, nous constatons que l'Europe demeure une importante source des cocitations.

Finalement, le Graphique $n^{\circ} 7$ correspond aux cocitations par genre. Ukraïnka, Blixen, De La Cruz, Woolf, Gordimer et Lispector sont 
LA PLACE DES ÉCRIVAINES DANS LA LITTÉRATURE MONDIALE:

CARTOGRAPHIE MÉTACRITIQUE DE LA MARGINALISATION DES FEMMES

principalement mises en relation avec des écrivains masculins. Frame, Djebar, Desai et Shikibu sont majoritairement cocitées avec d'autres femmes. Ainsi, il est difficile de tirer des conclusions sur les relations entre les genres des auteurs cocités.
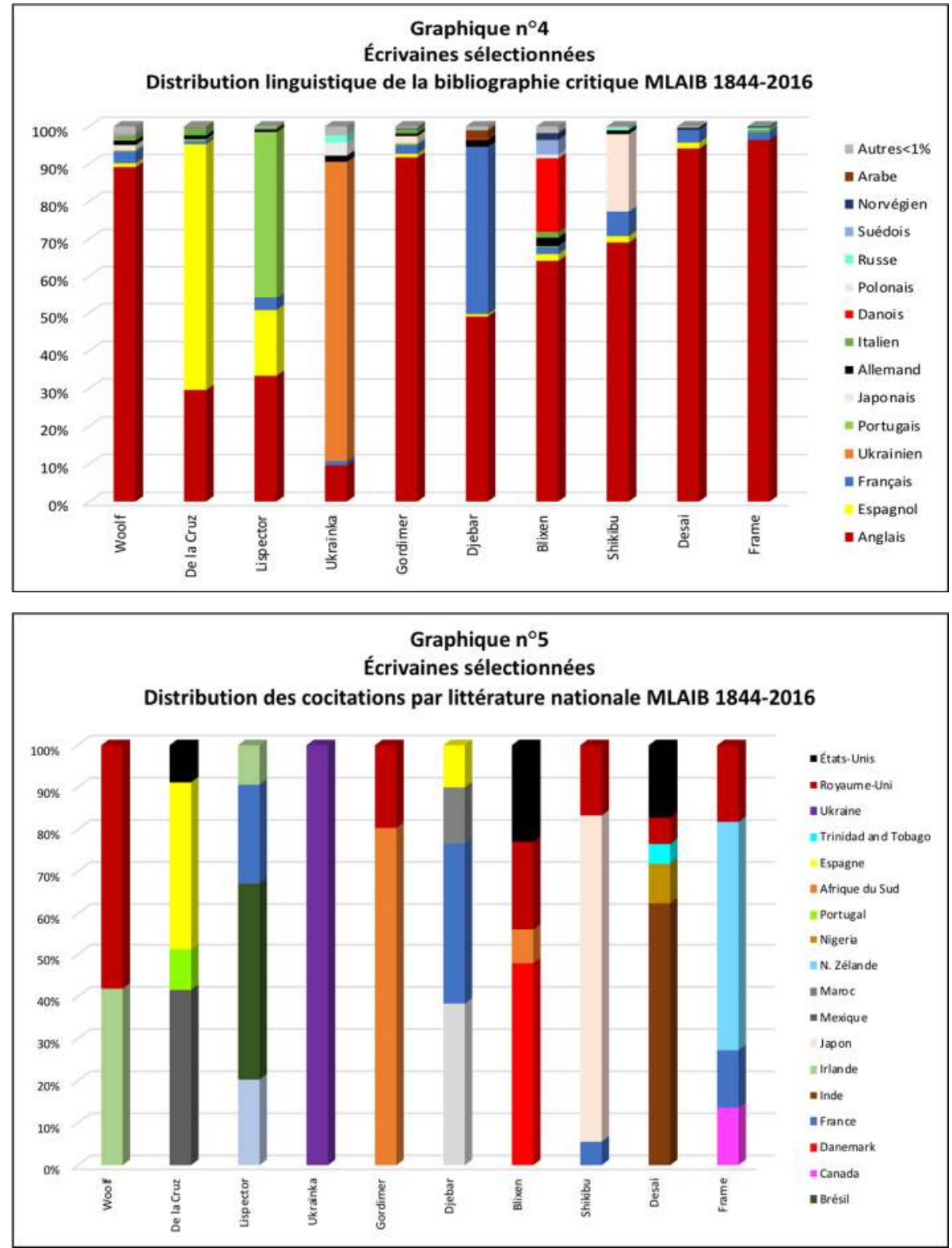

Pontos de Interrogaçầo, v. 9, n. 2, jul.-dez., p. 13-33, 2019. 

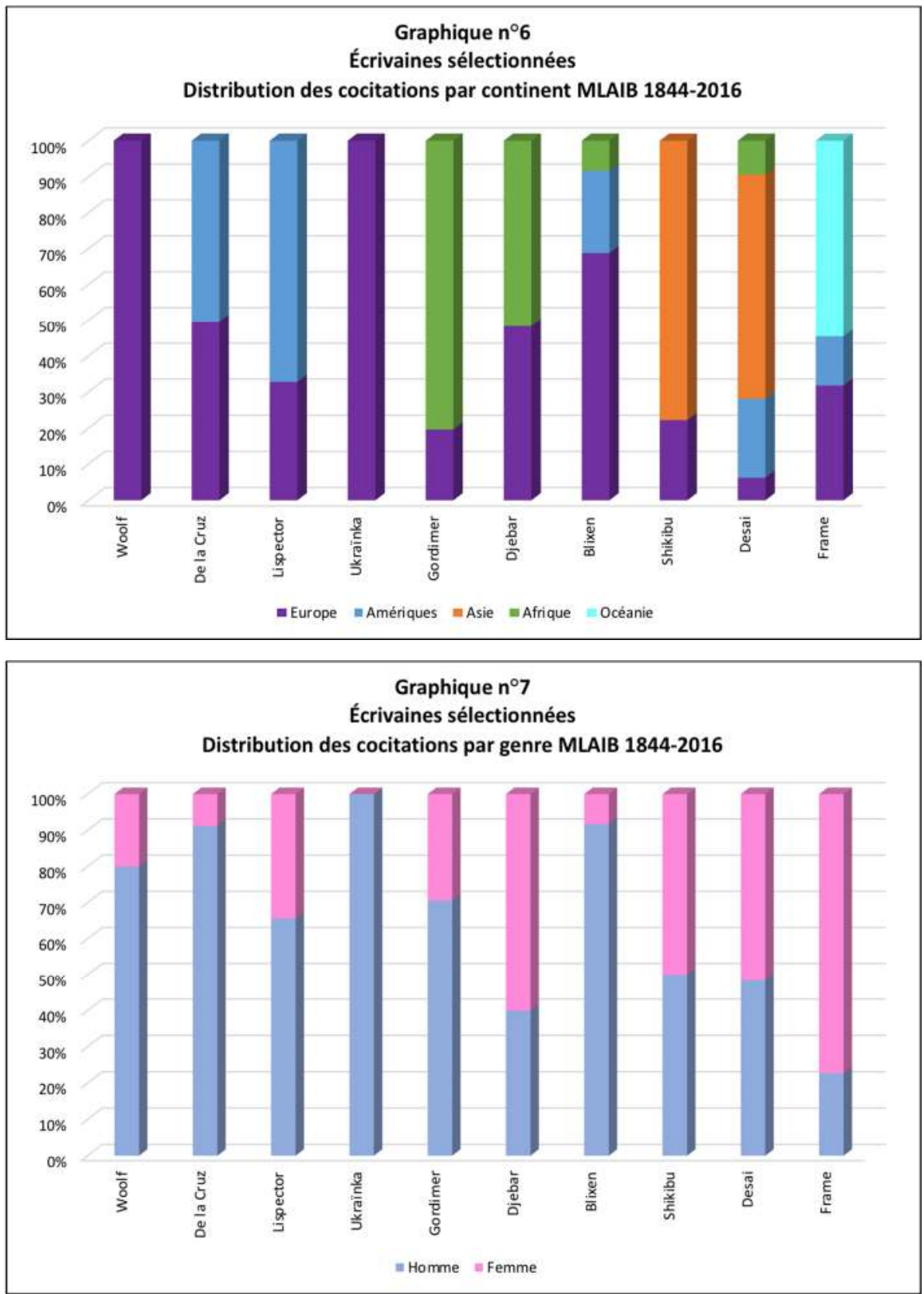

\section{Réflexions finales}

Grâce à l'analyse des métadonnées de la bibliographie critique contenue dans la base MLAIB, nous sommes en mesure de confirmer que les 
écrivaines occupent une place minoritaire dans la littérature mondiale. Elles sont nettement sous-représentées dans la majorité des littératures nationales, situation qui est très marquée en Europe. Spécifiquement, l'analyse métacritique de la bibliographie qui porte sur l'œuvre des 10 écrivaines sélectionnées nous permet de constater que les publications critiques montrent un biais vers l'anglais. Dans certains cas, nous observons que, malgré les difficultés qu'éprouvent les femmes européennes pour attirer l'attention de la critique, la plupart des auteurs cocités sont européens, montrant ainsi que le Vieux Continent continue d'être la principale référence en études comparées. Dans d'autres cas, les écrivains cocités sont presque exclusivement des compatriotes des écrivaines, dévoilant ainsi une circulation plutôt restreinte des œuvres signées par des femmes. Ces constats nous permettent d'affirmer que, selon nos recherches empiriques, les femmes occupent une place marginale dans la littérature mondiale, ainsi que dans la plupart des littératures nationales.

En conséquence, les résultats obtenus, qui reposent sur la loi des grands nombres et qui ont été générés par des méthodes vérifiables, confirment sans équivoque que la visibilité des femmes dans les études littéraires est extrêmement faible. Dans ce sens, comme le laisse entendre le personnage du roman de Wolitzer cité dans l'introduction de notre étude, les institutions littéraires constituent effectivement des boys' clubs. Vue ainsi, la mondialisation, tout du moins en études littéraires, semble ne concerner qu'une moitié de la population, ce qui nous fait douter de l'ouverture d'esprit dont elle se réclame.

\section{Références}

BOURDIEU, Pierre et LJD WACQUANT. Réponses. Pour une anthropologie réflexive. Paris: Seuil, 1992.

BOURDIEU, Pierre. Les règles de l'art. Genèse et structure du champ littéraire. Paris: Seuil, 1992.

BOURDIEU, Pierre. Méditations pascaliennes. Paris: Seuil, 1997. 
COZZENS, Susan E. "Using the Archive - Price, Derek Theory of Differences among the Sciences», Scientometrics 7, p. 431-41, 1985. Dissertation Abstracts International, http://proquest.com.

EVEN-ZOHAR, Itamar. «Polysystem Theory.» Poetics Today 11, p. 1-268, 1990.

FERRER, Carolina. "Les études littéraires à l'ère de la mondialisation: traces et trajets au prisme des nouveaux observables numériques », Zizanie 2.1, p. 76-101, 2018, https://www.zizanie.ca/les-etudes-litteraires-a-lere-de-lamondialisation.html.

FERRER, Carolina. «El boom hispanoamericano: del texto a la pantalla», In: Nuevas aproximaciones al cine hispánico: Migraciones temporales, textuales y étnicas en el bicentenario de las independencias iberoamericanas (1810-2010), Barcelona: Promociones y Publicaciones Universitarias, 2011, p. 79-101.

GENETTE, Gérard. Palimpsestes. La littérature au second degré. Paris: Seuil, 1982.

GLORIEUX, Frédéric. "Femmes de lettres, démographie (data.bnf.fr2017)», J'attends des résultats. Fouille de documents, expériences réussies et ratées, OpenEdition 2017, https://resultats. hypotheses.org/1048.

GUPTA, Suman. Globalization and Literature. Cambridge: Polity, 2009.

LANGLAIS, Pierre-Carl. "Les femmes ont-elles disparu de la littérature en 1830 ?", Sciences communes, 2017, https://scoms. hypotheses. org/824.

LEYDESDORFF, Loet. "Theories of Citation?" Scientometrics, no 43, p. 5-25, 1998.

Modern Language Association International Bibliography. www.mla.org.

NANCY Jean-Luc. La création du monde ou la mondialisation. Paris: Galilée, 2002.

PLANTÉ, Christine. "La place des femmes dans l'histoire littéraire : annexe, ou point de départ d'une relecture critique?», Revue d'histoire littéraire de la France 103.3, p. 655-668, 2003.

PRENDERGAST, Christopher (dir.). Debating World Literature. Londres: Verso, 2004.

PRICE, Derek de Solla. 1963. Little Science, Big Science. New York: Columbia University Press.

SAUSSY, Haun (dir.). Comparative Literature in an Age of Globalization. Baltimore: Johns Hopkins University Press, 2006. 
LA PLACE DES ÉCRIVAINES DANS LA LITTÉRATURE MONDIALE:

CARTOGRAPHIE MÉTACRITIOUE DE LA MARGINALISATION DES FEMMES

SMALL, Henry. 1973. "Co-citation in the Scientific Literature: A New Measure of the Relationship Between Two Documents.» Journal of the American Society for Information Science 24, p. 265-69.

The Wife, réal. Björn RUNGE, act. Jonathan PRYCE, Glenn CLOSE, 2017.

UNDERWOOD, Ted and David BAMMAN. "The Gender Balance of Fiction, 18002007), The Stone and the Shell, 2016, https://tedunderwood.com/2016/12/28/the-gender-balance-of-fiction-1800$2007 /$.

United Nations, http://data.un.org/Default.aspx.

VAN REES, Kees. "Modelling the Literary Field: From System-Theoretical Speculation to Empirical Testing», Canadian Review of Comparative Literature/Revue Canadienne de Littérature Comparée, March/mars, p. 91-101, 1997.

WOLITZER, Meg. The Wife. New York: Simon and Schuster, 2003.

ZHANG, Longxi. "The Changing Concept of World Literature». World literature in Theory. David DAMROSCH (dir.). Malden: Wiley Blackwell, 2014, p. 513-523.

Recebido em 27 de maio de 2019.

Aceito em 15 de junho de 2019. 